\title{
A Developed Real-time Tunnel Environment Monitoring System with C\#
}

\author{
Liu Baoshun \\ School of Civil and Environmental Engineering \\ University of Science and Technology Beijing, USTB \\ Beijing ,China \\ liubaoshun@ustb.edu.cn
}

\author{
Liu Yichao \\ School of Civil and Environmental Engineering \\ University of Science and Technology Beijing, USTB \\ Beijing, China \\ 814974846@QQ.com
}

\begin{abstract}
As China's mileage of Highway and urban transport tunnel extend speedily, some challenges to the safe operation of the tunnel occur. People pay more attention to the mechanical state of the traffic tunnel and the environmental problems caused by tunnels. Based on a new traffic tunnel in Henan province, the paper designed and developed a real-time monitoring system for the tunnel environment and pressure. Apparatuses such as sampling instruments, pressure transmitter, temperature sensor, wind speed sensor and the $\mathrm{CO}$ concentration transmission sensor were introduced. After analysis of collector KLM-4118 communication protocol, the system that displays the monitoring data with controls of Dundas Gauge vividly and visually can collect and analyze real-time monitoring data from tunnel and save them into SQL Server database after interpret the protocol. Now it has run steadily and offered great help to maintain the safety of the tunnel.
\end{abstract}

Keywords- tunnel environmental; monitoring; sampling instruments; Dundas Gauge component; database

\section{INTRODUCTION}

According to the length, highway tunnel can be divided into short tunnel $(\leq 500 \mathrm{~m})$, middle tunnel $(500 \mathrm{~m} \sim 1000 \mathrm{~m})$, long tunnel $(1000 \mathrm{~m} \sim 3000 \mathrm{~m})$ and extra-long tunnel $(>3000 \mathrm{~m})$ [1]. It is easy to encounter traffic accidents for long tunnel and extra-long tunnel, due to its high speed, high flow rate, poor lighting, poor air quality, environmental noise. Because the circular space is limited, tunnel accident is difficult to handle and it will take long time. In accordance with the international universal design standards, it is necessary to set up the environmental monitoring system that has been developed well in advanced western countries [2]. Tunnel environment monitor includes lining stress, ambient temperature, wind speed, CO concentration, and visibility [3]. With the development of highway in China, it is very important for the management of tunnels to gather real-time environment information from tunnels and display it with a friendly interface in the remote monitoring center. Some monitoring system has been developed such as Yan Qundong designed expressway tunnel monitoring system based on RSView32 [4]. The wireless sensor network has been set up for long tunnel by LeiXu [5]. With The combination of ZiGBee short distance wireless communication technology and GPRS, a design scheme of remote wireless monitoring system has been put forward to monitor tunnel environment [6].
A long tunnel in Henan, China, has left tunnel of $2955 \mathrm{~m}$ and right tunnel of $2960 \mathrm{~m}$. Its design speed is $80 \mathrm{~km} / \mathrm{h}$, and its net height is $5 \mathrm{~m}$. This paper has developed a real-time monitoring system for this tunnel to monitor its environment.

\section{MONITORING SYSTEM DESIGN}

The monitoring system is divided into tunnel sensor, monitoring station, the monitoring host computer, data processing computer, signal cable, signal optical cable, a remote monitoring center and other parts. Fig .1 is the topological structure of the real-time monitoring system.

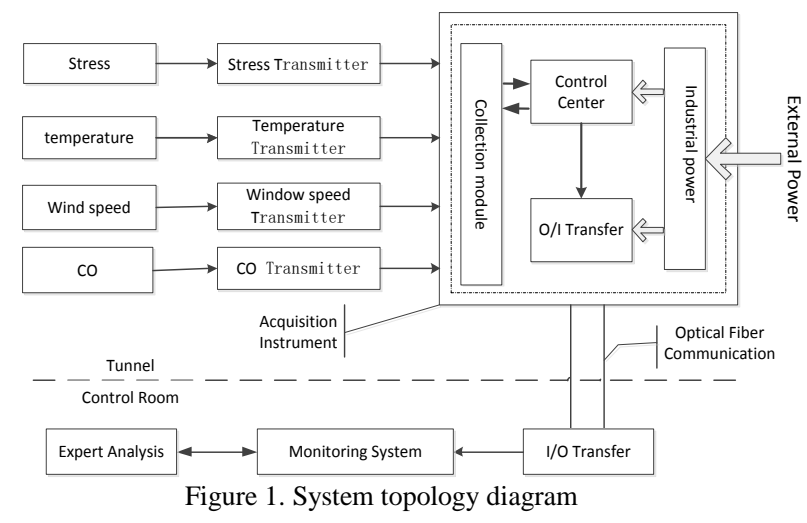

On acquiring data from tunnel, Data will be transferred to the control-room by optical fiber, so we need Optoelectrical converter(such as IR-1513S) to change signal between current signal and opto signal. If computers in control-room have no serial Port, the system needs another device to change ports between USB and RS-485.

\section{A. Sampling Instruments}

Data acquisition instruments KLM-4118 (Fig .2) made by Beijing Kunlun Seashore Sensor Technology Co. Ltd. are consisted of high quality units that ensure them to work steadily and safely. Many application such as sampling from rectification column[7] and hydraulic propeller static balance testing system [8].They are convenient to install and suitable to gather data from sensors and communicate with upper-position machines. 


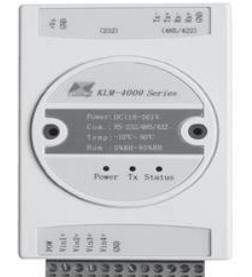

Figure 2. Acquisitioninstrument

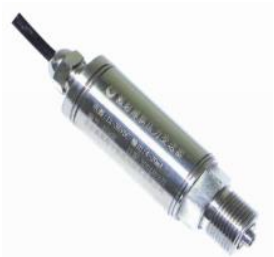

Figure 3. CYB-20S transmitter

Communication interface of KML-4118 that has 8 channels can be flexibly chosen between RS-232 and RS$485 / 422$. Period of its data refresh and alarm is $<0.5$ seconds. The input direct current signal is $4 \sim 20 \mathrm{~mA}$ and the power supply range is $18 \sim 36$ VDC. KML-4118 changes Standard current signal from detectors into digit signal between 0 to 9999 ,especially RS485 serial communication of RS485 can transfer long distance with strong anti-interference and higher transmission speed, so it is good to gather data with it [9]

\section{B. Tunnel Stress Monitoring Instrument}

To realize tunnel stress real-time warning and monitoring, stress monitoring system can gather stress data of the monitoring areas by borehole stress meters installed in the tunnel, and eventually generates cloud maps of tunnel wall stress contour that can help us find out where the high stress areas are and how its change trend.

CYB-20S(Fig .3) stress transmitter that is used in the system can transfer signal of stress to the data acquisition instrument KLM-4118. The instrument uses CYB-10S ion beam sputtered thin-film sensors as the stress sensor, and has integrated structure of electronic circuits. This transmitter is a stainless steel cylinder type structure, small size, convenient installation. It has high accuracy and stability, vibration and shock resistance, corrosion resistance and ability to withstand overload and interference. Its output is $4 \sim 20 \mathrm{~mA}$ and supply power is 24VDC.

\section{Temperature Monitoring Instrument}

SBWZ-K Temperature Instrument (Fig .4) is mainly responsible for gathering real-time temperature information. Micro-signal acquired from temperature sensor is amplified to 4 20mA standard signal and pass it into regional controller that can convert all the collected standard current signal to a standard protocol package 485 output signal.

SBWZ-K temperature sensor is two-wire transmission mode (power input and signal output share two common wire ). Its supply power is 24VDC.
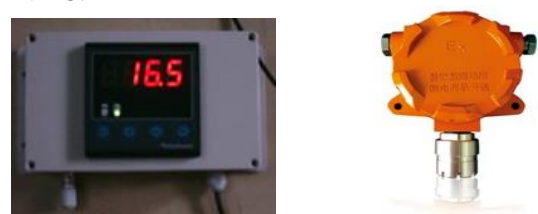

Figure 4. Temperature detector Figure 5. CO concentration detector

\section{CO Concentration detector}

HY-FM-02A CO Instrument (see Fig .5) is used in each tunnel monitoring sections to get real-time $\mathrm{CO}$ concentration information. Micro-signal acquired from $\mathrm{CO}$ sensor is amplified to $4 \sim 20 \mathrm{~mA}$ Standard signal and pass it into regional controller. The controller can convert current signal to 485 protocol signal as its output.

THY-FM-02A CO sensor uses advanced sensor technology in Germany, so there is a high degree of sensitivity of the CO gas concentration, low drift, long life span. Response time is fast and reliable, and explosionproof housing design. Instrument power supply:12-24V DC, maximum current of 22mA,range:0$1000 \mathrm{ppm}$,sensitivity: $1 \mathrm{ppm}$. The response time is less than $10 \mathrm{~s}$.

\section{E. Wind Speed Detector TF-Aland Opto-electrical converter}

Wind speed detector TF-A1(Fig .6) is mainly responsible for getting real-time wind speed information from monitoring points. Micro-signal from wind sensor are amplified to $4 \sim 20 \mathrm{~mA}$ standard signal, then pass it into regional controller. Regional controller converts the current signal to 485 protocol signal as its output.

TF-A1 is compact and lightweight, and it is easy to carry and assemble. Three cup on the sensor can make it acquire external environment information effectively. Its shell made of high quality aluminum alloy with external plating spray treatment, endows it with good anti-corrosion, anti-corrosion. The internal sliding bearing system ensures the accuracy of information. Its technical parameters: Precision $1 \mathrm{~m} / \mathrm{s}$ (response speed $0.2 \mathrm{~m} / \mathrm{s}$ ), range: $0 \sim 32.4$ $\mathrm{m} / \mathrm{s}$, power supply voltage: $7 \sim 24 \mathrm{~V}$ DC, signal output: $4 \sim 20 \mathrm{~mA}$ standard current signal.

IR-1513S Opto-electrical converter(Fig .7) with two $\mathrm{SC}$ interfaces is responsible for changing signal between current signal of RS-232/RS-422/RS-485 and opto signal.

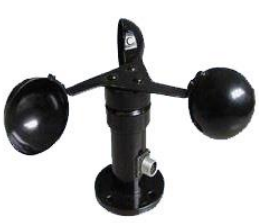

Figure 6 . Wind speed detector

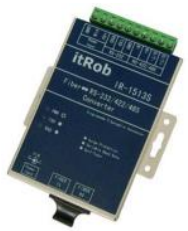

Figure 7. Opto-electrical converter

\section{F. Wind Speed Detector TF-Aland Opto-electrical converter}

Our monitoring system arranged 5 sections in the tunnel. Thinking of the geological exploration data, design of the tunnel pipeline, we fixed 3 stress sensors, 1 wind speed sensor, 1 temperature sensor and $1 \mathrm{CO}$ concentration sensor for each section(Fig .8 ). 


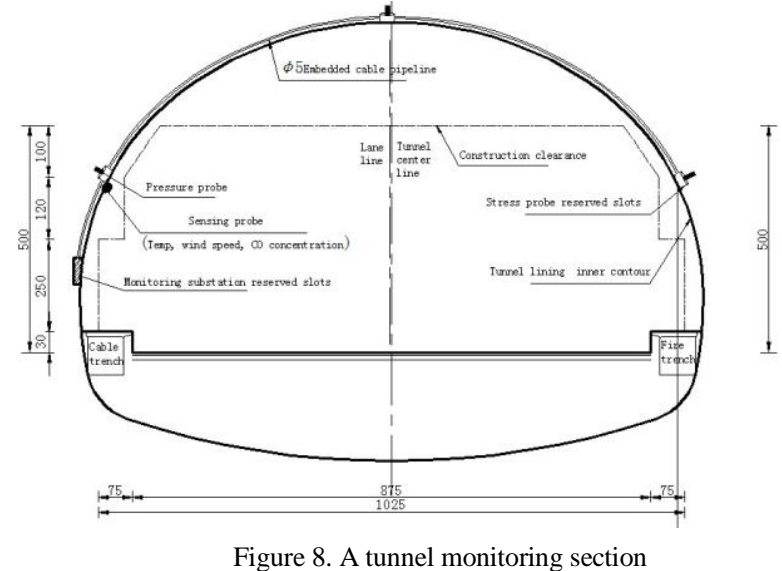

\section{COMMUNICATION PROTOCOLS OF KLM-4118}

KLM-4118 acquires environment information by sensors installed in the tunnel, but the information is shown as current signal, so it is necessary to decode it according to the input communication protocols of KLM4118.

All information input KLM-4118 from sensors is ASCII. Communication commands consist of the following parts:

(delimeter) (address) (command) (data) (checksum) (terminator)

delimeter: each command must start with a defined character, which is one of these characters: \#, \$,\%,@ ;

Address: It is a double-digit to specify the address of the target device;

Command: to specify a command use;

Data: data information of the command;

Checksum: checksum has two characters;

Terminator: each command must be ended with a carriage return;

Below is a command example to read analog data from KLM-4118:

\#0184

"\#”: a delimeter, it means that we request to read data;

" 01 ": address of the device;

" 84 ": used to check whether the accepted data is integrity;

After submitting the above command, we may receive the below answer:

$>-002503-000001-009999+000000-002501-002505-$

$002507+000000 \mathrm{~F} 3$

">": delimeter;

"F3":checksum;

The rest is each output data of 8 channels. When input of KLM-4118 is zero signal ( $4 \mathrm{~mA})$, the above channel output is 0 . If its input is full-signal (20A), the above channel output is 9999 . KLM-4118 can keep good linearity, so we can find out the corresponding relation between input and output easily.

\section{DEVELOPMENT OF MONITORING SYSTEM}

Based on VS2008 platform, C\# language was used to develop the system and SQL Server2008 was used as DBMS to build up monitoring database. Tunnel environment real- time monitoring system can display, alarm and store the monitor information. The main function is as follows:

(1)Real-time displays and stores the tunnel environmental state of each monitoring section, including wall stress, temperature, wind speed and $\mathrm{CO}$ concentration;

(2)Varied trend of environment parameters could be displayed;

(3)Distribution curves of the tunnel environment are real-time drawn;

The following describes the data acquisition module of the system

\section{A. Establishment of the Database}

Sql Server 2008 is used to store data. Fig .9 is one table to define sensors and Fig .10 is used to store monitoring data.

\begin{tabular}{|c|c|c|c|}
\hline \multicolumn{4}{|c|}{ WIN-FM1OMUAGGKD...g-dbo.SenseSet } \\
\hline & COLUMN_NAME & DATA_TYPE & IS_NULLABLE \\
\hline & CanNo & nchar(10) & v \\
\hline & SenseName & nchar(10) & v \\
\hline 8 & id & int & 回 \\
\hline
\end{tabular}

Figure 9. Structure of channel table

\begin{tabular}{|c|c|c|c|}
\hline \multicolumn{3}{|c|}{ WIN-FM1OMUAGGK...onitoringValue* } & \multirow{2}{*}{$\begin{array}{l}\text { WIN-FM1OM } \\
\text { IS_NULLABLE }\end{array}$} \\
\hline & COLUMN_NAME & DATA_TYPE & \\
\hline & MonitoringTime & datetime & $\square$ \\
\hline & CanNo & nchar (10) & 回 \\
\hline & MonitoringData & bigint & $\nabla$ \\
\hline & MonitoringResult & decimal $(18,3)$ & 四 \\
\hline & munit & nchar(15) & 目 \\
\hline 8 & id & bigint & 圆 \\
\hline
\end{tabular}

Figure 10. Structure of monitoring table

\section{B. The Realization of the Serial Communication Technology}

The communication is mainly completed by the SerialPort class provided by C\# [10]. After modifying parameters (see Fig .11), we have allocated the port number and channels where each sensor is connected to KLM-4118.

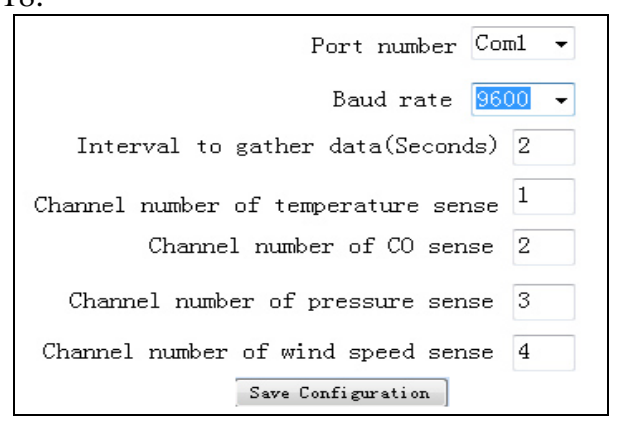

Figure 11. Sets the sensor's port

\section{1) Receive Data in Control-Room}

We can receive data by below programs: private void timer1_Tick(object sender, EventArgs e)

\{ string codeString = "\#0184\r";

try $\{$ serialPort 1.Write(codeString); \}

catch (Exception ex) \{

timer1.Enabled = false; 
MessageBox.Show("serialPort isnot open, failture to receive data ! |r" + ex.ToString(), "alert", MessageBoxButtons.OK, MessageBoxIcon.Warning); ..... \{\}

The request period to send data is controlled by setting Timer control's interval in Fig .11. Below are parts of programs to receive data:

private void serialPort1_DataReceived(object sender, System.IO.Ports.SerialDataReceivedEventArgs e)

\{

string acceptMsg = serialPort1.ReadTo("|r"); textBox1.Invoke(new EventHandler(delegate

\{

textBox1.Text+= acceptMsg;

Acceptdata(acceptMsg);// procedure to decode and store data to database

dataGridViewX1.DataSource $=$ null;

dataGridViewX1.DataSource

$=$

myDb.ReturnDs("select * from monitoringValue order by monitoringtime desc");

\})); \}

2) Decoding the Received Data

Because KLM-4118 keeps good linearity, after identifying the received string we can calculate its input current intensity according the below formula:

$\mathrm{ma}=($ number $/ 9999) * 16+4$

number: extracted data from received string;

ma: input current intensity(Ma).

Then we need to calculate the measured value of each sensor that is connected to different channels. Below programs have realized it.

public double CalculateMonitoringValue(double number, string ChannelType)

$\mathrm{ma}=($ number $/ 9999) * 16+4$

double realValue $=0$;

switch (ChannelType) \{

case "1":// temperature sensor

realValue $=(25.0 / 4.0) * \mathrm{ma}-75 ;$

break;

case "2"://CO

realValue $=62.5 *(\mathrm{ma}-4.0)$;

break;

case " $3 ": / /$ stress

realValue $=5.0 / 16.0 *$ ma $-5.0 / 4.0$;

break;

case " $4 ": / /$ wind speed

realValue $=\mathrm{ma} * 2.025-8.10$;

break; \}

return realValue;

\section{Display Data Received from KLM-4118 in Control- Room}

According to protocols of KLM-4118, after received data are decoded, they can be displayed in DataGridViewX(Fig .12).To make a vivid show, the system also offers the ability to show data in Gauge controls (Fig .13).

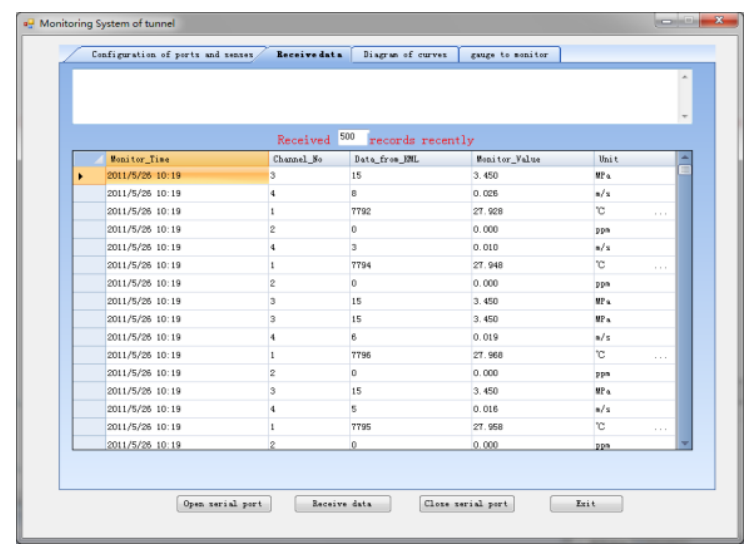

Figure 12. Display data with DataGridViewX

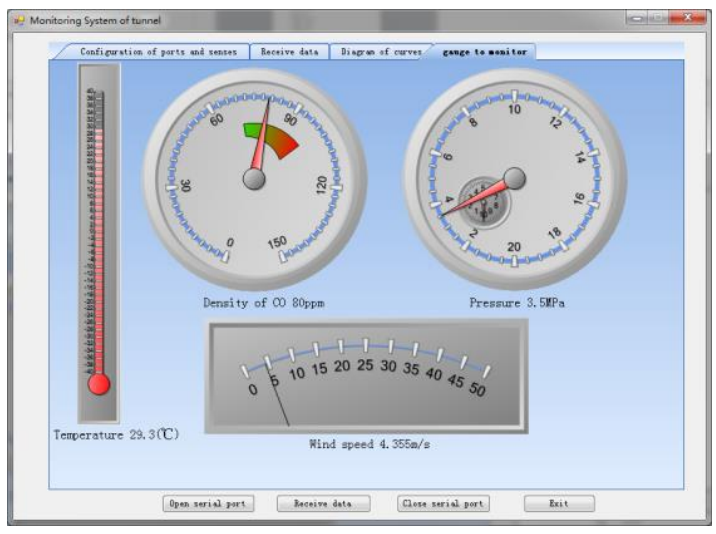

Figure 13. Show data with gauge

\section{CONCLUSIONS}

The paper introduces a way to monitor tunnel environment including stress state of the section wall, temperature, wind speed and CO concentration in the tunnel. Real-time received data can be stored into database and shown with Gauge controls rather than others show data only in text. Since the system has been set up, it keeps running steadily. It is beneficial to increase the ability to manage the tunnel.

\section{REFERENCES}

[1] People's Republic of China traffic,Code for design of highway tunnel (JTGD70-2004). Beijing: People transport press,2004.

[2] Lou Cheng, "Environment Mornitoring and Control \&Management Development Tendency of ExpressWay Tunnel," Northern Communications,vol.5,2012,pp.122-123.

[3] Zhao Qi,XU jun,Yu Jiayan,Bao Lei,Zhang Weibin,Li Danian, "Long tunnel investigation of air quality," Environment and Ecology in the Three Gorge,vol.33,January,2011,pp.14-16

[4] Yan Qundong,Li Xiaojiang, Wang Qian,Jin Chaohui, “The design and implement of the tunnel supervision and control system based on RSView32 software, " Technology and Economy in Areas of communications, vol.45,2008,pp.63-65

[5] Lei $\mathrm{xu}, \mathrm{Li}$ yang,Li sihui, "Design and implementation of environment monitoring system for long tunnel based on WSN, "Compter Engineering and Design, vol.34(8),Aug.2013,pp.2676-2679.

[6] Zhang Zhen hai,Dang Jian wu,Min Yong Zhi, "Design of wireless monitoring system for railway tunnel environment based on wireless sensor network,"Journal of Lanzhou Jiaotong University,Vol.33,Aug.2014,pp.40-44,doi:10.3969/j.issn.10014373.2014.04.009. 
[7] Guo,Hao Huang,Hua, "The design of remote data sampling and controlling distributed system," Microcomputer Information, vol.22,2006,pp.137-138.

[8] Hu Hongbo,Liu Zhiyong,Wang Cuntang, "Research on Computer Testing System for Hydraulic Propeller Static Equilibrium,"Fluid Power Transmission and Control,July 2010,pp.13-15.
[9] Rao Jie,Huang Hua, "Realization of a distributed monitoring system based on serial port communication for temperature and pressure of exact rectification," Electric Drive, Vol.36,2006,pp.4749.Zheng Eqi, "SQL Server practical course," Beijing:Electronic Industry Press, 2009.

[10] Andrew Troelsen, "Pro C\# 2010 and the .NET 4 Platform," Beijing:Post and Telecom Press,2011. 